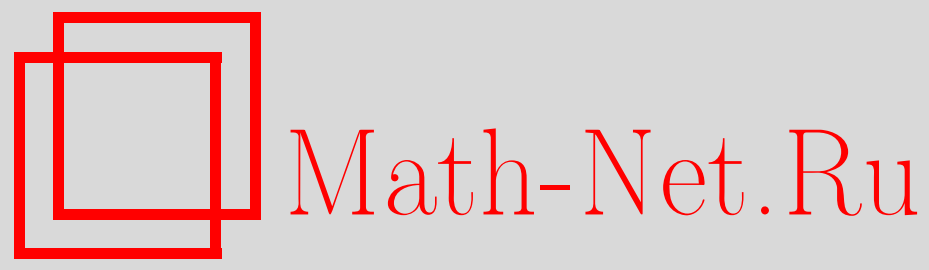

В. В. Рыжиков, Полиморфизмы, джойнинги и тензорная простота динамических систем, Функи. анализ и его прил., 1997, том 31, выпуск 2, 45-57

DOI: https://doi.org/10.4213/faa462

Использование Общероссийского математического портала MathNet.Ru подразумевает, что вы прочитали и согласны с пользовательским соглашением

http://www . mathnet.ru/rus/agreement

Параметры загрузки:

IP : 18.207 .199 .55

26 апреля 2023 г., 16:56:02

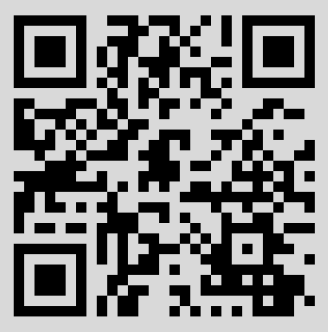




\title{
Полиморфизмы, джойнинги и тензорная простота динамических систем
}

\author{
(c) 1997. В. В. Рыжиков
}

\section{$\S 1$. Введение}

В статье рассматриваются сохраняющие меру $\mu$ действия $\left\{T_{g}: g \in G\right\}$ группы $G$ на пространстве Лебега $(X, \mathscr{B}, \mu), \mu(X)=1$, называемые динамическими системами, и группы унитарных операторов $\left\{T_{g}\right\}$ на $L_{2}(\mu):\left(T_{g} f\right)(x)=f\left(T_{g}(x)\right)$, индуцированных этими действиями. Преобразование $T_{g}$ множества $X$ и отвечающий ему оператор называются автоморфизмами и обозначаются одним и тем же символом.

Оператор $P: L_{2}(\mu) \rightarrow L_{2}\left(\mu^{\prime}\right)$ называется марковским (или бистохастическим), если $P$ и $P^{*}$ переводят неотрицательные функции в неотрицательные, а постоянную функцию в себя. Марковский оператор $P$ связан с мерой $\nu$ на $X \times X:$

$$
\forall f, f^{\prime} \in L_{2}(\mu) \quad\left\langle P f, f^{\prime}\right\rangle=\int f \otimes f^{\prime} d \nu .
$$

Такие меры называется полиморфизмами (порядка 2), они введены в [3] (см. там же о соответствии между полиморфизмами и марковскими операторами). Полиморфизм порядка $n$ есть мера на $n$-кубе $X^{n}=X \times \cdots \times X$ ( $n$ сомножителей), проекции которой на сомножители совпадают с $\mu$. (Произведение $n$ экземпляров меры $\mu$ обозначается через $\mu^{n}$; оператор, отвечающий мере $\mu \otimes \mu$, обозначается через $\Theta$ ).

Джойнингом набора автоморфизмов $T_{1}, \ldots, T_{n}$ называется полиморфизм порядка $n$, инвариантный относительно $T_{1} \otimes \cdots \otimes T_{n}$. О теории джойнингов и ее приложениях см. недавний обзор [14].

Мы говорим, что полиморфизм $\nu$, заданный на $X^{n}$, принадлежит классу $M(m, n), n>m>1$, если его проекции на $m$-мерные грани декартова $n$-куба совпадают с мерой $\mu^{m}$. Если для действия $\left\{T_{g}\right\}$ мера $\mu^{n}$ является единственной мерой класса $M(m, n)$, инвариантной относительно $T_{g} \otimes \cdots \otimes T_{g}$, то мы говорим, что это действие принадлежит классу $S(m, n)$ или обладает свойством $S(m, n)$.

Тензорно простым называется действие класса $S(3,4)$. Авторы статьи $[7]$ ввели в рассмотрение класс $P I D=\bigcap_{n>2} S(2, n)$ (их результат: класс $P I D$ замкнут относительно декартовых произведений). В [9] доказано, что свойство $S(2,4)$ влечет за собой $S(2, n)$ для всех $n>4$. Как будет видно, $S(2 p-1,2 p)=$ $S(2,4)$ для всех $p>1$. Таким образом, класс тензорно простых действий совпадает с классом PID. Словосочетание «тензорная простота» подчеркивает, что 
система и ее тензорная степень не имеют нетривиальных марковских сплетений (формальные определения см. ниже).

Открыты вопросы о совпадении классов $S(2,3)$ и $S(3,4)$ и существовании слабо перемешивающего $\mathbb{Z}$-действия с нулевой энтропией, не принадлежащего классам $S(n, n+1)$. Значительный интерес к этим задачам связан с тем, что в классе $S(3,4)$ давняя проблема Рохлина о кратном перемешивании имеет положительное решение (см., например, $[6,9])$.

1.1. Пример $\mathbb{Z}$-действия класса $S(3,4)$. Используя метод аппроксимаций [8], несложно построить автоморфизм $T$, удовлетворяющий следующему условию: для некоторой последовательности $k(i) \rightarrow \infty$

$$
\forall A, B \quad \mu\left(T^{k(i)} A \cap B\right) \rightarrow \frac{1}{2} \mu(A \cap B)+\frac{1}{2} \mu(T A \cap B),
$$

что эквивалентно слабой сходимости

$$
T^{k(i)} \rightarrow Q=\frac{1}{2} I+\frac{1}{2} T .
$$

(Из эргодичности такого автоморфизма $T$ следует, что он обладает свойством слабого перемешивания: найдется последовательность $m(i) \rightarrow \infty$, такая, что $T^{m(i)} \rightarrow \Theta$.)

Пусть $\nu$ - некоторый эргодический джойнинг класса $M(3,4)$. Рассмотрим оператор $P: L_{2}(\mu \otimes \mu \otimes \mu) \rightarrow L_{2}(\mu)$, соответствуюший мере $\nu$ :

$\forall f_{1}, f_{2}, f_{3}, f \in L_{2}(\mu) \quad\left\langle P\left(f_{1} \otimes f_{2} \otimes f_{3}\right), f\right\rangle=\int_{X \times X \times X \times X} f_{1} \otimes f_{2} \otimes f_{3} \otimes f d \nu$.

Отметим, что из проекционных свойств меры $\nu$ вытекает, что $P(f \otimes g \otimes h)$ является постоянной функцией, если постоянна одна из функций $f, g, h$. Из инвариантности меры $\nu$ относительно $T \otimes T \otimes T \otimes T$ вытекает условие сплетения

$$
T P=P(T \otimes T \otimes T) ;
$$

следовательно,

$$
T^{k} P=P(T \otimes T \otimes T)^{k}, \quad Q P=P(Q \otimes Q \otimes Q) .
$$

Теперь получаем

$$
\begin{aligned}
\frac{1}{2}(I+T) P & =\frac{1}{2} P(I \otimes I \otimes I)+\frac{1}{2} P(T \otimes T \otimes T)=P(Q \otimes Q \otimes Q) \\
& =\frac{1}{8}(P(I \otimes I \otimes I)+P(I \otimes T \otimes T)+\cdots+P(T \otimes T \otimes T)) .
\end{aligned}
$$

Приняв во внимание эргодичность меры $\nu$ (эквивалентную неразложимости оператора $P$ в сумму двух различных сплетающих марковских операторов), получаем

$$
P=P(T \otimes I \otimes I) \quad \text { или } \quad P=P(T \otimes T \otimes I) .
$$

Так как $T^{m(i)} \rightarrow \Theta$, то в первом случае

$$
P(f \otimes g \otimes h)=P(\Theta f \otimes g \otimes h)=\Theta f \Theta g \Theta h,
$$

что влечет за собой

$$
\nu=\mu \otimes \mu \otimes \mu \otimes \mu
$$


(второй случай приводит к аналогичному заключению). Таким образом, автоморфизм $T$ является тензорно простым.

Отметим, что этот результат можно получить также из глубокой теоремы [6]: автоморфизм с сингулярным спектром является тензорно простым.

1.2. Кратное перемешивание и джойнинги. Мы говорим, что преобразование $T$ перемешивает с кратностью $k$, если для любых $A_{0}, \ldots, A_{k} \in \mathscr{B}$ при $\left|z_{m}-z_{n}\right| \rightarrow \infty, 0 \leqslant m<n \leqslant k$,

$$
\mu\left(T^{z_{0}} A_{0} \cap T^{z_{1}} A_{1} \cap \cdots \cap T^{z_{k}} A_{k}\right) \rightarrow \mu\left(A_{0}\right) \mu\left(A_{1}\right) \cdots \mu\left(A_{k}\right) .
$$

Известно (см., например, [6, 9]), что для перемешивающего автоморфизма $T$ в предположении, что он не перемешивает с кратностью $k$, найдется мера $\nu \neq \mu^{k+1}$ класса $M(2, k+1)$ и последовательности $z_{0}(i), \ldots, z_{k}(i) \rightarrow \infty$, такие, что

$$
\mu\left(T^{z_{0}(i)} A_{0} \cap T^{z_{1}(i)} A_{1} \cap \cdots \cap T^{z_{k}(i)} A_{k}\right) \rightarrow \nu\left(A_{0} \times A_{1} \times \cdots \times A_{k}\right) .
$$

Мера $\nu$, конечно, инвариантна относительно $T \otimes \cdots \otimes T(k+1$ сомножителей).

Если же для перемешивающего автоморфизма $T$ таких нетривиальных джойнингов (отличных от $\mu^{k+1}$ ) нет, то $T$ является перемешивающим порядка $k$.

Опишем приложение тензорной простоты и инварианта типа кратного перемешивания к задаче симметрии прошлого и будущего динамической системы. Примеры слабо перемешивающих автоморфизмов, не сопряженных своему обратному, были построены в [11]. Представляют интерес явные и простые инварианты, которые отличают некоторые автоморфизмы от их обратных.

Для некоторой последовательности $n(i) \rightarrow \infty$ и числа $a>0$ найдется автоморфизм $T$ с простым сингулярным спектром, такой, что для некоторой меры $\nu \in M(2,3)$ выполнено следующее условие:

$$
\forall A, B, C \in \mathscr{B} \quad \liminf _{i \rightarrow \infty} \mu\left(A \cap T^{n(i)} B \cap T^{3 n(i)} C\right) \geqslant a \nu(A \times B \times C),
$$

причем

$$
\mu\left(A \cap T^{-n(i)} A \cap T^{-3 n(i)} A\right) \rightarrow \mu(A)^{2} .
$$

(Конструкция такого автоморфизма $T$ ввиду ограниченности объема статьи не приводится.)

Из сказанного непосредственно следует, что $T$ и $T^{-1}$ неизоморфны. Так как из [6] вытекает, что автоморфизм $T$ является тензорно простым, получим $\nu=\mu \otimes \mu \otimes \mu$. Таким образом, на паре фиксированных последовательностей $\{n(i)\},\{3 n(i)\}$ автоморфизм $T$ обладает свойством частичного кратного перемешивания:

$$
\liminf _{i \rightarrow \infty} \mu\left(A \cap T^{n(i)} B \cap T^{3 n(i)} C\right) \geqslant a \mu(A) \mu(B) \mu(C),
$$

а его обратный этим свойством не обладает.

В 2 мы изложим фрагмент элементарной теории тензорной простоты, а в $\S \S 3,4$ установим тензорную простоту для некоторых классов динамических систем. 


\section{§2. Некоторые следствия тензорной простоты}

Приведем определение тензорной простоты в терминах операторов. Пусть $H$ - пространство функций из $L_{2}(X, \mu)$ с нулевым средним. Действие $\left\{T_{g}: g \in G\right\}$ принадлежит классу $S(3,4)$, если существует единственный марковский оператор $J: L_{2}(\mu) \rightarrow L_{2}(\mu \otimes \mu \otimes \mu)$, удовлетворяюший условиям

$$
J H \subset H \otimes H \otimes H \quad \text { и } \quad J T_{g}=\left(T_{g} \otimes T_{g} \otimes T_{g}\right) J \quad \forall g \in G .
$$

Этим условиям (при любом $T_{g}$ ) удовлетворяет тривиальный оператор, образ которого (по определению) есть одномерное пространство постоянных функций.

Назовем внутренним иентрализатором действия $T_{g}^{\otimes n}=T_{g} \otimes \cdots \otimes T_{g}$ ( $n$ сомножителей) множество всех марковских операторов $J$, коммутирующих с $T_{g}^{\otimes n}$, таких, что полиморфизмы, отвечающие этим операторам, принадлежат классу $M(2 n-1,2 n)$. Эквивалентное определение: внутренний централизатор действия $T_{g}^{\otimes n}$ состоит из таких марковских операторов $J$, что для любого набора марковских операторов $P_{1}, \ldots, P_{n}: L_{2} \rightarrow L_{2}$, один из которых равен $\Theta$, выполняется соотношение

$$
J\left(P_{1} \otimes \cdots \otimes P_{n}\right)=\left(P_{1} \otimes \cdots \otimes P_{n}\right) J=\Theta \otimes \cdots \otimes \Theta .
$$

ПРЕДЛОЖЕНИЕ 2.1. (а) Тензорная простота влечет за собой свойство $S(m, n)$ для всех $n>m>1$.

(b) Для любого $n$ тензорная простота действия $T_{g}$ равносильна тривиальности внутреннего чентрализатора действия $T_{g}^{\otimes n}$.

ДокАЗАТЕльство. (а) Покажем, что $S(3,4)$ влечет за собой $S(4,5)$ (общий случай рассматривается аналогично). Пусть действие $T_{g}$ не принадлежит классу $S(4,5)$. Найдется отличная от $\mu \otimes \mu \otimes \mu$ мера $\nu \in M(2,3)$, инвариантная относительно $T_{g} \otimes T_{g} \otimes S_{g}$, где действие $S_{g}$ изоморфно действию $T_{g} \otimes T_{g} \otimes T_{g}$. (Пространство $(X, \mathscr{B}, \mu)$ изоморфно всем пространствам вида $(X, \mathscr{B}, \mu) \times \cdots \times$ $(X, \mathscr{B}, \mu)$.) Пусть оператор $P: L_{2} \otimes L_{2} \rightarrow L_{2}$ отвечает полиморфизму $\nu$, т. е.

$$
\forall f_{1}, f_{2}, h \in L_{2}(\mu) \quad\left\langle P\left(f_{1} \otimes f_{2}\right), h\right\rangle=\int\left(f_{1} \otimes f_{2} \otimes h\right) d \nu .
$$

Для оператора $J$, связанного с $P$ соотношением

$$
\forall f_{1}, f_{2}, f_{1}^{\prime}, f_{2}^{\prime} \in L_{2}(\mu) \quad\left\langle J f_{1}, f_{2} \otimes f_{1}^{\prime} \otimes f_{2}^{\prime}\right\rangle=\left\langle P\left(f_{1} \otimes f_{2}\right), P\left(f_{1}^{\prime} \otimes f_{2}^{\prime}\right)\right\rangle,
$$

выполнены условия (1), что легко проверяется. Так как $T_{g} \in S(3,4)$, получим, что $J$ тривиален (ему отвечает мера $\mu \otimes \mu \otimes \mu \otimes \mu$ ). Тогда тривиален оператор $P$. Действительно, $\left\langle P\left(f_{1} \otimes f_{2}\right), P\left(f_{1} \otimes f_{2}\right)\right\rangle=0$, если $\int f_{1} d \mu=0$; значит, $P\left(f_{1} \otimes f_{2}\right) \equiv 0$. Мера $\nu$, соответствующая оператору $P$, будет равна $\mu \otimes \mu \otimes \mu$. Получили противоречие.

(b) Пусть нетривиальный внутренний оператор $J$ сплетает $T_{g} \otimes T_{g} \otimes T_{g}$ и $T_{g}$. Тогда $J^{*} J$ - нетривиальный внутренний оператор, коммутирующий с $T_{g} \otimes T_{g} \otimes T_{g}$. Для $n=3$ п. (b) доказан. Теперь определим оператор $P$ :

$$
\left\langle P\left(f_{1} \otimes \cdots \otimes f_{5}\right), f_{6}\right\rangle=\left\langle J^{*} J\left(f_{1} \otimes f_{2} \otimes f_{3}\right), J^{*} J\left(f_{4} \otimes f_{5} \otimes f_{6}\right)\right\rangle .
$$


Проверяется, что $P^{*} P$ - нетривиальный внутренний оператор, коммутирующий с $T_{g} \otimes T_{g} \otimes T_{g} \otimes T_{g}$. Если $P^{*} P$ тривиален, то тривиален и $P$. Для $n=4$ п. (b) доказан.

Рассуждая аналогичным образом, получим, что из тривиальности внутреннего централизатора действия $T_{g} \otimes \cdots \otimes T_{g}$ вытекает тензорная простота действия $T_{g}$.

Рассмотрим случай расширений динамической системы, сохраняющих свойство кратного перемешивания.

ПРЕДЛОЖЕНИЕ 2.2. Пусть $S$ перемешивает $c$ кратностью $k$, а косое произведение $R, R(x, y)=\left(S(x), T_{x}(y)\right)$, является перемешиваюшим. Если все преобразования $T_{x}$ коммутируют с некоторым тензорно простьм действием $\Psi$ (быть может, некоммутативным), то косое произведение $R$ перемешивает с кратностью $k$.

ДокАЗАтЕльство. Рассмотрим случай $k=2$. Обозначим через $\lambda$ меру $\mu \otimes \mu$, которая инвариантна относительно $R$. Пусть для некоторых последовательностей $z_{0}, z_{1}, z_{2} \rightarrow \infty$ и некоторой меры $\nu \in M(2,3)$ (класс $M(2,3)$ рассматривается здесь относительно пространства $(X \times Y, \lambda))$

$$
\lambda\left(R^{z_{0}} A_{0} \cap R^{z_{1}} A_{1} \cap T^{z_{2}} A_{2}\right) \rightarrow \nu\left(A_{0} \times A_{1} \times A_{2}\right) .
$$

Мера $\nu$ задана на произведении $(X \times Y) \times(X \times Y) \times(X \times Y)$, которое нам удобно представить в виде $(X \times X \times X) \times(Y \times Y \times Y)$. Из того, что $S$ перемешивает кратно, вытекает, что проекция меры $\nu$ на $(X \times X \times X)$ совпадает с $\mu \otimes \mu \otimes \mu$. Меру $\nu$ разложим в систему условных мер $\left\{\nu_{w}: w \in X \times X \times X\right\}$ :

$$
\nu\left(A_{1} \times A_{2} \times A_{3} \times B_{1} \times B_{2} \times B_{3}\right)=\int_{A_{1} \times A_{2} \times A_{3}} \nu_{w}\left(B_{1} \times B_{2} \times B_{3}\right) d w .
$$

Для почти всех $w$ верно, что $\nu_{w} \in M(2,3)$. Так как $R$ коммутирует с $\Phi=I \otimes \Psi$, мера $\nu$ инвариантна относительно $\Phi \otimes \Phi \otimes \Phi$. Теперь получаем, что условные меры $\nu_{w} \in M(2,3)$ инвариантны относительно $\Psi \otimes \Psi \otimes \Psi$. Но $\Psi$ есть тензорно простая система; следовательно, почти все $\nu_{w}$ равны $\mu \otimes \mu \otimes \mu$. Таким образом, мы получили, что $\nu=\lambda \otimes \lambda \otimes \lambda$. Тем самым свойство кратного перемешивания порядка $k=2$ установлено. Случай $k>2$ рассматривается аналогично; при $k>3$ нужно также применить предложение 2.1 .

Понятие тензорной простоты близко по смыслу к свойству дизъюнктности динамических систем: две системы $T$ и $S$ дизъюнктны (в смысле Фюрстенберга), если $\mu \otimes \mu-$ единственный джойнинг систем $T$ и $S$.

Эквивалентное определение: две системы (стохастически) дизъюнктны, если они имеют единственное марковское сплетение $\Theta$ - оператор, образ которого есть одномерное пространство постоянных функций.

Классический пример: орициклический и геодезический потоки дизъюнктны (хотя они унитарно эквивалентны), что вытекает из результатов о К-свойстве геодезического потока [1], о равенстве нулю энтропии орициклического потока [5] и теоремы Фюрстенберга о дизъюнктности К-системы и действия с нулевой энтропией (см. [9]). Последняя теорема следует из теоремы Пинскера: для любой динамической системы каждый К-фактор и фактор Пинскера независимы. Действительно, пусть $\nu$ - джойнинг $T$ и $S$, где $T$ есть К-автоморфизм, а $S$ имеет 
нулевую энтропию. Тогда у динамической системы $(T \times S, X \times X, \nu)$ координатные факторы (а они изоморфны $S$ и $T$ ) независимы; следовательно, $\nu=\mu \otimes \mu$.

Со свойством тензорной простоты непосредственно связана давняя задача Фюрстенберга: если $S$ и $T$ дизъюнктны, будут ли дизьюнктны $S$ и $T \otimes T ?$ Следующие утверждения демонстрируют эту связь и дополняют результаты [9].

ПрЕДЛОЖЕНИЕ 2.3. Если автоморфизм $S$ или автоморфизм $T$ является тензорно простым и $S$ и $T$ дизъюнктны, то $S$ и $T \otimes T$ также дизъюнктньл.

ДокаЗАтЕльство. Если оператор $P$ сплетает $T \otimes T$ и $S$, то оператор $P^{*} P$ сплетает $T \otimes T$ с $T \otimes T$. Из дизъюнктности $T$ и $S$ вытекает, что

$$
P(f \otimes \mathbb{I})=P(\mathbb{I} \otimes f)=\text { Const }=\Theta f .
$$

Легко видеть, что оператору $P^{*} P$ отвечает мера $\eta \in M(3,4)$, инвариантная относительно $T^{\otimes 4}$. Если автоморфизм $T$ является тензорно простым, то $\eta=\mu^{4}$ и операторы $P^{*} P$ и, следовательно, $P$ тривиальны.

Если же $S$ является тензорно простым, для аналогичной цели нужно рассмотреть оператор $Q$, сплетающий $S \otimes T$ и $R=T$ и удовлетворяющий условию $Q(f \otimes \mathbb{I})=Q(\mathbb{I} \otimes f)=$ Const. Оператор $Q^{*} Q$ сплетает $S \otimes T$ с $S \otimes T$. Теперь определим оператор $P$ :

$$
\left\langle P\left(f_{1} \otimes f_{3}\right), f_{2} \otimes f_{4}\right\rangle=\left\langle Q\left(f_{1} \otimes f_{2}\right), Q\left(f_{3} \otimes f_{4}\right)\right\rangle .
$$

Оператор $P^{*} P$ является внутренним и сплетает $S \otimes S$ с $S \otimes S$. В силу свойства тензорной простоты автоморфизма $S$ оператор $P^{*} P$ тривиален, а следовательно, тривиален $Q$. Таким образом, $T \otimes T$ и $S$ дизьюнктны.

Доказательство следующего утверждения несложно получить из результатов [9].

ПРЕДЛОЖЕНИЕ 2.4. Пусть $\left\{T_{i}: i \in C\right\}$ - семейство попарно дизъюнктных тензорно простых преобразований, и пусть $\prod_{i \in D} T_{i}$ изоморфен $\prod_{i \in D^{\prime}} T_{j}$, где $D, D^{\prime} \subset C$. Тогда $D=D^{\prime}$.

\section{§3. Некоторые тензорно простые системы}

Тензорно простым является (любой) перемешивающий унипотентный поток на однородном пространстве $X=G / \Gamma$ конечного объема. Как будет показано далее, это утверждение вытекает из результатов [12]. М. Ратнер доказала, что любой эргодический джойнинг унипотентного потока является гладким: он сосредоточен на гладком подмногообразии $Y \subset X^{n}\left(X^{n}-\right.$ декартова степень пространства $X$ ) и абсолютно непрерывен относительно меры Лебега на $Y$. Назовем потоки с таким свойством S-потоками.

В [12] доказано больше: эргодическим джойнингом унипотентного потока является $H$-инвариантная мера, сосредоточенная на замкнутой орбите $H x \subset$ $X^{n}$ связной подгруппы $H$ группы $G^{\otimes n}$. А. Н. Старков, используя этот факт, решил проблему о кратном перемешивании для однородных потоков [13]. Доказательство в [13] можно модифицировать: после того как проведена редукция к случаю унипотентных потоков, достаточно доказать следующее утверждение. 
ТЕорема 3.1. Слабо перемешиваюший S-поток является тензорно проcmblм.

СЛЕДСТВИЕ. Унипотентный перемешивающий поток является тензорно простьм и перемешиваюшим всех степеней.

ДокАЗАтЕльство. Как было показано в 2 , свойство $S(3,4)$ эквивалентно свойству $S(2 p-1,2 p)$ для любого $p>1$. Пусть размерность пространства $X$ равна $d$ и на нем действует $\mathrm{S}$-поток $\left\{T_{t}\right\}$. Покажем, что поток принадлежит классу $S(n, n+1)$ при $n>d$ (из этого следует тензорная простота потока $\left.\left\{T_{t}\right\}\right)$.

Фиксируем $n>d$ и предположим, что $\nu$ - эргодическая относительно $\left\{T_{t} \otimes \cdots \otimes T_{t}\right\}(n+1$ сомножителей) мера класса $M(n, n+1)$. Предположим, что $\nu$ сосредоточена на гладком многообразии $Y \subset X^{n+1}, \operatorname{dim}(Y)<\operatorname{dim}\left(X^{n+1}\right)$. Для некоторого фиксированного $\delta>0$ рассмотрим множества

$$
V_{r}=\bigcup_{0<t_{1}, \ldots, t_{r}<\delta}\left(T_{t_{1}} \otimes \cdots \otimes T_{t_{r}} \otimes I \otimes I \otimes \cdots\right) Y .
$$

Так как множество $V_{r}$ инвариантно относительно эргодического потока $T_{t}^{\otimes n+1}$ (поток $T_{t}$ является слабо перемешивающим относительно $\mu$ ), мера (объем) множества $V_{r}$ равна 0 или 1 . Последнее невозможно, если $\delta$ достаточно мало.

Таким образом, считаем, что $\mu^{n+1}\left(V_{r}\right)=0$, а следовательно, $\operatorname{dim}\left(V_{r}\right)<$ $\operatorname{dim}\left(X^{n+1}\right)$. Начиная с некоторого $r$, размерности множеств $V_{r}$ стабилизируются: $\operatorname{dim}\left(V_{r}\right)=\operatorname{dim}\left(V_{r+1}\right)$. Это означает, что для достаточно малых $s_{1}, \ldots, s_{r}$, $s, s>0$,

$$
\left(T_{s_{1}} \otimes \cdots \otimes T_{s_{r}} \times T_{s} \otimes I \otimes I \otimes \cdots\right) Y \subset V_{r} .
$$

Переходя от многообразий к джойнингам, определим меры

$$
\begin{gathered}
\nu_{\left(t_{1}, \ldots, t_{n+1}\right)}\left(A_{1} \times \cdots \times A_{n+1}\right)=\nu\left(T_{-t_{1}} A_{1} \times \cdots \times T_{-t_{n+1}} A_{n+1}\right), \\
\bar{\nu}=\int_{0<t_{i}<\delta} \nu_{\left(t_{1}, \ldots, t_{r}, 0,0, \ldots\right)} d t_{1} \cdots d t_{r} .
\end{gathered}
$$

При достаточно малом $s>0$ получим, что мера $\bar{\nu}$ и ее сдвиг

$$
\left(I_{(1)} \otimes \cdots \otimes I_{(r)} \otimes T_{s} \otimes I_{(r+2)} \otimes I_{(r+3)} \cdots \otimes I_{(n+1)}\right) \bar{\nu}
$$

имеют общую часть, так как обе эти меры абсолютно непрерывны относительно меры Лебега на $V_{r}$, а $s$ мало. Поскольку разложение этих мер на эргодические компоненты известно (это сдвиги эргодической меры $\nu$ ), получим

$$
\nu_{\left(s_{1}, \ldots, s_{r}, s, 0,0, \ldots\right)}=\nu_{\left(t_{1}, \ldots, t_{r}, 0,0,0, \ldots\right)}
$$

(слева и справа выписаны эргодические компоненты). Таким образом,

$$
\nu=\left(T_{s_{1}-t_{1}} \otimes \cdots \otimes T_{s_{r}-t_{r}} \otimes T_{s} \otimes \cdots \otimes I \otimes I \otimes \cdots\right) \nu .
$$

Но из того, что мера $\nu$ принадлежит классу $M(n, n+1)$ и инвариантна относительно $\left(\cdots \otimes T_{s} \otimes \cdots \otimes I \otimes \cdots\right)(n+1$ сомножителей $)$, где $T_{s}$ - слабо перемешивающий автоморфизм, следует $\nu=\mu^{n+1}$ (см. конец $\S 3$ и доказательство 
леммы 4.4). Таким образом, для достаточно больших $n$ мера $\mu^{n+1}$ - единственная $\left(T_{t} \otimes \cdots \otimes T_{t}\right)$-инвариантная мера класса $M(n, n+1)$. Следовательно, поток является тензорно простым.

Действия с почти простым централизатором. Будем говорить, что действие $\Psi$ имеет почти простой марковский централизатор $M C(\Psi)$, если для некоторого $a>0$ найдется конечный набор операторов $\left\{Q_{i}\right\}, Q_{i} \in M C(\Psi)$, удовлетворяющих условиям:

- $Q_{i}^{*} Q_{i}>a I, Q_{i} Q_{i}^{*}>a I$

- каждый неразложимый оператор $J \neq \Theta, J \in M C(\Psi)$, имеет вид $J=$ $Q_{m} R$, где $R$ - автоморфизм, коммутирующий с действием $\Psi$.

Неизвестно, является ли тензорно простым перемешивающее действие $\left\{T^{i}\right.$ : $i \in \mathbb{Z}\}$, марковский централизатор которого есть множество выпуклых сумм операторов $T^{i}$ и оператора $\Theta$. (В теории джойнингов это свойство обозначается через MSJ(2).) Известно, что для слабо перемешивающих потоков MSJ(2) влечет за собой тензорную простоту, а для дискретных действий эта задача еще не решена.

Напомним, что автоморфизм $T$ частично перемешивает с показателем $a>0$, если

$$
\forall A, B \quad \liminf _{i} \mu\left(A \cap T^{i} B\right) \geqslant a \mu(A) \mu(B) .
$$

Рангом автоморфизма $T$ называется минимальное натуральное число $r$, такое, что некоторая последовательность разбиений $\xi_{j}$ вида

$$
\xi_{j}=\left\{C_{1}^{j}, T C_{1}^{j}, T^{2} C_{1}^{j}, \ldots, T^{h_{1}(j)} C_{1}^{j}, \ldots, C_{r}^{j}, T C_{r}^{j}, T^{2} C_{r}^{j}, \ldots, T^{h_{r}(j)} C_{r}^{j}, Q_{j}\right\}
$$

стремится к разбиению на точки (заметим, что тогда $\mu\left(Q_{j}\right) \rightarrow 0$ ). (Если такого $r$ нет, говорят, что $T$ имеет бесконечный ранг.)

Частично перемешивающие (с показателем $0<a<1) \mathbb{Z}^{n}$-действия конечного ранга служат примерами динамических систем, для которых выполнены условия следующего утверждения.

ТЕОРема 3.2. Почти простое действие, иентрализатор которого содержит слабо перемешиваюший не перемешиваюший автоморфизм, является тензорно простьлм.

Марковский сплетающий оператор назовем неразложимым, если ему отвечает эргодическая мера (для оператора, сплетающего $T$ и $S$, мера эргодична относительно $T \otimes S)$.

ЛеммА 3.3. Пусть $J_{i}, i \in \mathbb{N}$, - неразложимые марковские операторы, сплетаюиие почти простое действие $\left\{T_{g}: g \in G\right\}$ с его тензорньм кубом. Если $J_{0}^{*} J_{i} \neq \Theta$ для всех $k \neq 0$, то для некоторых $z, w \in \mathbb{N}$ найдется автоморфизм $R$, коммутирующий $с T_{g}$, такой, что вылолнено равенство $J_{w}=J_{z} R$.

Доказательство леммы 3.3 является несложной модификацией доказательства теоремы 2 из [4].

Доказательство теоремы 3.2 проводится по следующей схеме. Пусть внутренний оператор $J: L_{2} \rightarrow L_{2} \otimes L_{2} \otimes L_{2}$, сплетающий $\Psi$ и $\Psi \otimes \Psi \otimes \Psi$, является неразложимым. 
Предположим, что $J$ нетривиален: $J \neq J \Theta$. Покажем, что для некоторой последовательности $k(i) \rightarrow \infty$ последовательность операторов

$$
J_{i}=\left(S^{k(i)} \otimes I \otimes I\right) J
$$

удовлетворяет условиям леммы 3.3 .

Пусть $S^{m(i)} \rightarrow D \neq \Theta$. Тогда $D-$ марковский оператор, коммутирующий с действием $\Psi$. Так как $S^{-m(j)} \rightarrow D^{*}$, то

$$
S_{i}=S^{m(i)-m(j(i))} \rightarrow D^{*} D \neq \Theta
$$

для некоторой последовательности $j(i)$. Так как действие $\Psi$ почти простое, оператор $D$ имеет вид $\sum_{k} a_{k} P_{k} Q_{k}$, где $P_{k}$ - операторы, являющиеся средними по некоторым мерам на групповом централизаторе $C(\Psi)$ действия $\Psi$. При $a>0$ выполняется неравенство

$$
D^{*} D>a \sum_{k} a_{k}^{2} P_{k}^{*} P_{k}>a\left(\int_{C(\Psi)} R d \gamma_{i}(R)\right)^{*}\left(\int_{C(\Psi)} R d \gamma_{i}(R)\right)
$$

( $\gamma_{i}$ - некоторая нормированная мера на централизаторе). Следовательно,

$$
J^{*}\left(D^{*} D \otimes I \otimes I\right) J>a J^{*}\left(\left(\int_{C(\Psi)} R d \gamma_{i}(R)\right)^{*}\left(\int_{C(\Psi)} R d \gamma_{i}(R)\right) \otimes I \otimes I\right) J,
$$

причем последний оператор, что несложно доказать, будет нетривиальным. Так как

$$
J^{*}\left(S_{i} \otimes I \otimes I\right) J \rightarrow J^{*}\left(D^{*} D \otimes I \otimes I\right) J \neq \Theta,
$$

для достаточно больших $i$ имеем

$$
J^{*}\left(S_{i} \otimes I \otimes I\right) J \neq \Theta .
$$

По лемме 3.3

$$
J=\left(S_{i} \otimes I \otimes I\right) J R .
$$

Так как слабое замыкание степеней автоморфизма $S_{i}$ содержит оператор $\Theta$, то для некоторого оператора $Q$ из слабого замыкания множества $\left\{R^{n}: n>0\right\}$ получим соотношение

$$
J=(\Theta \otimes I \otimes I) J Q=J \Theta,
$$

т.е. оператор $J$ тривиален.

\section{§4. Некоторые расширения, сохраняющие тензорную простоту}

Если косое произведение имеет вид $R(x, y)=\left(S(x), T^{n(x)}(y)\right)$, где $T$ есть бернуллиевский сдвиг, то кроме очевидного $S$-фактора преобразование $R$ обладает непрерывным семейством факторов. Действительно, пусть $\mathscr{A}$ есть $\sigma$-алгебра, инвариантная относительно $T$; тогда $\mathscr{B} \times \mathscr{A}$ инвариантна относительно $R$. Для бернуллиевского $T$ можно указать непрерывное семейство разбиений $\left\{\xi_{h}\right.$ : $h \in(0, h(T))\}$, где $\xi_{h}$ - образующее разбиение для фактора с энтропией, равной $h$. Отметим, что энтропия тензорно простого преобразования $R$ равна 
нулю (см. [7]), а последнее выполнено, когда $\int n(x) d \mu=0$ и энтропия автоморфизма $S$ равна нулю. Сказанное выше и следующая теорема позволяют строить тензорно простые системы с непрерывными семействами факторов.

Теорема 4.1. Пусть $R, T$ - перемешивающие преобразования, где $R$ есть косое произведение над $S$ следующего вида:

$$
R(x, y)=\left(S(s), T^{n(x)}(y)\right), \quad \int n(x) d \mu=0 .
$$

(a) Если $S$ принадлежит классу $S(p-1, p)$, то $R$ также принадлежит классу $S(p-1, p)$;

(б) если $S$ перемешивает с кратностью $k$, то $R$ также перемешивает с кратностью $k$.

Теорема 4.1 вытекает из следующего утверждения (см. также доказательство предложения 2.2).

Tеорема 4.2. Пусть $\mathscr{C}: X^{n} \rightarrow M(n-1, n)$. Если $\left(S, T^{f(x)}\right)-$ перемешивающее косое произведение, где $T$ - перемешивающее преобразование и $\int f d \mu=0$, то уравнение

$$
\mathscr{C}\left(S\left(x_{1}\right), \ldots, S\left(x_{n}\right)\right) \equiv\left(T^{f\left(x_{1}\right)} \otimes \cdots \otimes T^{f\left(x_{n}\right)}\right) \mathscr{C}\left(x_{1}, \ldots, x_{n}\right)
$$

имеет единственное решение $\mathscr{C}\left(x_{1}, \ldots, x_{n}\right) \equiv \mu^{n}$.

Доказательство проведем после вспомогательных утверждений.

Цилиндрическим каскадом называем отображение $(S, f):(X \times \mathbb{Z}) \rightarrow(X \times \mathbb{Z})$, определенное соотношением

$$
(S, f)(x, a)=(S(x), a+f(x)) \quad \forall x \in X, \forall a \in \mathbb{Z},
$$

где $S$ - преобразование множества $X$, а $f: X \rightarrow \mathbb{Z}$ - некоторая функция.

ТЕОРема 4.3. Пусть $S$ есть эргодическое преобразование и для функиии $f: X \rightarrow \mathbb{Z}$ выполнено условие $\int f(x) d \mu=0$. Тогда

(а) иилиндрический каскад $(S, f):(X \times \mathbb{Z}) \rightarrow(X \times \mathbb{Z})$ являетсл консервативной системой;

(b) дяя почти каждого $x \in X$ найдется бесконечная последовательность натуральньх чисел $\left\{q_{i}(x)\right\}$, такал, что $q_{0}(x)=0, q_{i}(x)<q_{i+1}(x)$ и для любого $i$

$$
\sum_{n=0}^{q_{i}(x)-1} f\left(S^{n}(x)\right)=0 .
$$

Доказательство теоремы см. в $[10,2]$; п. (b) вытекает непосредственно из (а) и означает, что множество $\left\{N: \sum_{n=0}^{N} f\left(S^{n}(x)\right)=0\right\}$ бесконечно для почти всех $x \in X$. (Отметим, что теорема, аналогичная п. (b), выполнена для потоков [15].)

Лемма 4.4. Пусть $T$ перемешивает и задана мера $\nu \in M(n, n+1)$. Если хотя бы одна из последовательностей $m_{1}(i), \ldots, m_{n}(i)$ стремится $\kappa$ бесконечности при $i \rightarrow \infty$, то имеет место сходимость

$$
\nu_{i}:=\left(I \times T^{m_{1}(i)} \times \cdots \times T^{m_{n}(i)}\right) \nu \rightarrow \mu^{n+1} .
$$


Доказательство проведем для случая $n=2$. Рассмотрим оператор $P: L_{2}^{\otimes 2} \rightarrow$ $L_{2}$, такой, что

$$
\langle P(A \otimes B), C\rangle=\nu(A \times B \times C) .
$$

Предположим для определенности, что $m_{1}(i) \rightarrow \infty$. Нам нужно доказать, что

$$
P_{i}:=\left(T^{m_{1}(i)} \otimes T^{m_{2}(i)}\right) P \rightarrow P \Theta \quad(i \rightarrow \infty)
$$

$\left(\Theta\right.$ - ортопроектор на пространство констант в $\left.L_{2}(\mu)\right)$. Для некоторой последовательности $i^{\prime}$ имеем

$$
\left(T^{m_{1}\left(i^{\prime}\right)} \otimes T^{m_{2}\left(i^{\prime}\right)}\right) P \rightarrow(\Theta \otimes Q) P=(\Theta \otimes \Theta) P
$$

где равенство возникает из-за того, что оператор $P$ внутренний. Предельному оператору $(\Theta \otimes \Theta) P=P \Theta$ отвечает мера $\mu^{3}$. Так как мере $\nu_{i}$ соответствует оператор $P_{i}$, получим $\nu_{i^{\prime}} \rightarrow \mu^{3}$. Отсюда вытекает, что $\nu_{i} \rightarrow \mu^{3}$, так как выбор сходящейся подпоследовательности $\nu_{i^{\prime}}$ был произвольным.

Лемма 4.5. Если $\left(S, T^{f(x)}\right)$ перемешивает, то при $i \rightarrow \infty$ для любого $K$ мера множества $\{x:|F(x, i)|<K\}$ стремится $\kappa 0$.

ДокАЗАТЕЛьСтво. Рассмотрим множество вида

$$
U=X \times \bigcup_{-N<i<N} T^{i} Y
$$

где $T^{i} Y$ - непересекающиеся множества, а число $N$ выбрано так, чтобы выполнялось $a-2(K / N)>2 \tilde{\mu}(U)>0$. Требуемое множество $Y$ всегда найдется (для построения можно воспользоваться известной леммой Рохлина-Халмоша).

Предположим, что для некоторой последовательности $i^{\prime} \rightarrow \infty$ будет выполнено неравенство

$$
\mu\left(\left\{x:\left|F\left(x, i^{\prime}\right)\right|<K\right\}\right)>a>0
$$

Тогда

$$
\lim _{i \rightarrow \infty} \tilde{\mu}\left(U \cap R^{i} U\right)>a \tilde{\mu}(U)-2(K / N)>2 \tilde{\mu}(U) \tilde{\mu}(U),
$$

что противоречит свойству перемешивания автоморфизма $R$.

ДоКАЗАТЕЛЬСТво теоРЕмы 4.2. Для простоты рассмотрим случай $n=3$. Зададим метрику на множестве $M(2,3)$. Пусть некоторое семейство $\left\{B_{i}\right\}_{i \in \mathbb{N}}$ всюду плотно в $\mathscr{B}$ и задана биекция $\sigma: \mathbb{N}^{3} \rightarrow \mathbb{N}$. Определим расстояние между мерами $\nu$ и $\eta$ следующим образом:

$$
\rho(\nu, \eta)=\sum_{i, j, k} 2^{-\sigma(i, j, k)}\left|\nu\left(B_{i} \times B_{j} \times B_{k}\right)-\eta\left(B_{i} \times B_{j} \times B_{k}\right)\right| .
$$

Положим

$$
F(x, i)=\sum_{n=0}^{i} f\left(S^{n-1}(x)\right)
$$

Для всех $i$

$$
\mathscr{C}\left(S^{i}(x), S^{i}(y), S^{i}(z)\right) \equiv\left(T^{F(x, i)} \otimes T^{F(y, i)} \otimes T^{F(z, i)}\right) \mathscr{C}(x, y, z) .
$$


Из эргодичности $S \otimes S \otimes S$ вытекает, что почти всюду в $X \times X \times X$ либо $\mathscr{C}(x, y, z) \neq \mu^{3}$, либо $\mathscr{C}(x, y, z) \equiv \mu^{3}$. Пусть выполнено первое условие; тогда для некоторого $c>0$ мера тех $(x, y, z)$, для которых $\rho\left(\mathscr{C}(x, y, z), \mu^{3}\right)>c$, больше, чем $c$. Для некоторых множества $A, \mu(A)>0$, и числа $b>0$ выполнено условие

$$
\mu \otimes \mu\left(\left\{(y, z): \rho\left(\mathscr{C}(x, y, z), \mu^{3}\right)>c\right\}\right)>b \quad \forall x \in A .
$$

Заметим, что для почти всех точек из $A$ множество $\left\{i^{\prime}: S^{q_{i^{\prime}}(x)}(x) \in A\right\}$ бесконечно. Это вытекает из того, что преобразование $U(x)=S^{q_{1}(x)}(x)$ (при условии, что число $q_{1}(x)>0$ выбирается минимальным для всех $x$ ) обратимо почти всюду, а следовательно, сохраняет меру и для него выполняется свойство возвращаемости: для почти всех $x$ множество $\left\{i^{\prime}: U^{i^{\prime}}(x) \in A\right\}$ бесконечно. Приходим к противоречию с предположением (4.1). Действительно, в силу теоремы 4.3 имеем (здесь мы обозначаем $i^{\prime}$ через $i$ )

$$
\mathscr{C}\left(S^{q_{i}(x)}(x), S^{q_{i}(x)}(y), S^{q_{i}(x)}(z)\right) \equiv\left(I \otimes T^{F\left(y, q_{i}(x)\right)} \otimes T^{F\left(z, q_{i}(x)\right)}\right) \mathscr{C}(x, y, z) .
$$

Из лемм 4.4 и 4.5 при $x \in A$ получим, что

$$
\mu \otimes \mu\left(\left\{(y, z): \rho\left(\mathscr{C}\left(S^{q_{i}(x)}(x), S^{q_{i}(x)}(y), S^{q_{i}(x)}(z)\right), \mu^{3}\right)>c\right\}\right) \rightarrow 0 .
$$

Таким образом, для почти всех $(x, y, z)$

$$
\mathscr{C}\left(S^{q_{i}(x)}(x), S^{q_{i}(x)}(y), S^{q_{i}(x)}(z)\right) \rightarrow \mu \otimes \mu \otimes \mu ;
$$

следовательно,

$$
\mathscr{C}(x, y, z) \equiv \mu \otimes \mu \otimes \mu .
$$

Автор благодарен Б. М. Гуревичу, В. И. Оселедцу и А. М. Степину за консультации.

\section{ЛитеРАТУРА}

1. Аносов Д. В. Геодезические потоки на римановых многообразиях отрицательной кривизны. Труды МИАН, 90 (1967).

2. Atkinson G. Reccurence of co-cycles and random walks. J. London Math. Soc., 13, 486-488 (1976).

3. Вершик A. М. Многозначные отображения с инвариантной мерой (полиморфизмы) и марковские процессы. Зап. науч. сем. ЛОМИ, 72, 26-62 (1977).

4. Glasner E., Host B., Rudolph D. Simple systems and their higher order self-joinings. Israel J. Math., 78, 131-142 (1992).

5. Гуревич Б. М. Энтропия потока орициклов. ДАН СССР, 136, №4, 768-770 (1961).

6. Host B. Mixing of all orders and pairwise independent joinings of systems with singular spectrum. Israel J. Math., 76, 289-298 (1991).

7. del Junco A., Rudolph D. On ergodic action whose self-joinings are graphs. Ergodic Theory Dynamical Systems, 7, 531-557 (1987).

8. Каток А. Б., Степин А. М. Аппроксимации в эргодической теории. УМН, 22, вып. 5, 81-106 (1967).

9. King J. Ergodic properties where order 4 implies infinite order. Israel J. Math., 80, 65-86 (1992). 
10. Крыгин А. Б. Пример цилиндрического каскада с аномальными метрическими свойствами. Вестник МГУ, сер. 1, №5, 26-32 (1975).

11. Оселедеи В. И. Две неизоморфные динамические системы с одинаковым простым непрерывным спектром. Функц. анализ и его прил., 5, вып. 3, 75-79 (1971).

12. Ratner $M$. On Raghunathan's measure conjecture. Ann. Math., 134, 545-607 (1991).

13. Старков A. Н. О кратном перемешивании однородных потоков. ДАН, 333. 28-31 (1993).

14. Thouvenot J.-P. Some properties and applications of joinings in ergodic theory. In: Ergodic Theory and Its Connections with Harmonic Analysis: Proceedings of the Alexandria 1993 Conference, K. E. Petersen and I. A. Salama, eds., LMS Lecture Note Series 205, 1995, pp. 207-235.

15. Шнейберг И. Я. Нули интегралов вдоль траекторий эргодических систем. Функц. анализ и его прил., 19, вып. 2, 92-93 (1985).

Московский государственный университет им. М. В. Ломоносова 25 января 1996 г. 